\title{
Análise Estatística do Algoritmo LMS Filtrado Modificado
}

\author{
Juan R. V. López, Orlando J. Tobias e Rui Seara
}

\begin{abstract}
Resumo-Este artigo apresenta um modelo analítico para o algoritmo LMS filtrado modificado (MFxLMS). O algoritmo MFxLMS é uma versão modificada do algoritmo FxLMS convencional, visando melhorar a velocidade de convergência para aplicações de controle ativo de ruído e vibrações. Modelos analíticos para o momento de primeira ordem e curva de aprendizagem do filtro adaptativo são derivados. Esses modelos são obtidos sem invocar a teoria da independência, que é uma suposição clássica para a modelagem de algoritmos LMS. Os modelos aqui derivados consideram a hipótese de adaptação lenta. Resultados de simulação Monte Carlo ratificam a qualidade de predição do comportamento do algoritmo a partir do modelo proposto, utilizando tanto sinais de entrada brancos quanto coloridos.
\end{abstract}

Palavras-chave-Adaptação lenta, algoritmo LMS, algoritmo MFxLMS.

Abstract-This paper presents a statistical analysis of the modified filtered-x least-mean-square (MFxLMS) algorithm. The MFxLMS algorithm is a modified version of the standard FxLMS aiming to improve the convergence speed for active noise control and vibration applications. Analytical models for the first moment of the adaptive filter weights and learning curve are derived. These models are obtained without invoking the independence theory, which is a classical assumption for the modeling of LMS algorithms. The models here derived allow for a slow adaptation condition. Results of Monte Carlo (MC) simulations show a very good agreement with those obtained from the proposed model for both white and colored Gaussian input data.

Keywords-LMS algorithm, MFxLMS algorithm, slow adaptation condition.

\section{INTRODUÇÃO}

O controle ativo de ruído acústico e vibrações é uma das mais importantes aplicações do algoritmo LMS filtrado (FxLMS) [1], [2]. O FxLMS é uma modificação do algoritmo LMS convencional, que leva em consideração o efeito do caminho secundário (denotado por $\mathbf{s}$ na Fig. 1). Tal modificação consiste em filtrar o sinal de referência com um filtro idêntico ao do caminho secundário (caso ideal). No entanto, na prática, apenas uma estimativa de s, representada

Juan R. V. López e Rui Seara, LINSE-Laboratório de Circuitos e Processamento de Sinais, Depto. de Eng. Elétrica, Universidade Federal de Santa Catarina, Florianópolis, SC, E-mails: \{juan, seara\}@linse.ufsc.br.

Orlando J. Tobias, LINSE/UFSC e Departamento de Engenharia Elétrica e Telecomunicações, Universidade Regional de Blumenau, SC, E-mail: tobias.oj@ieee.org.

Este trabalho foi parcialmente financiado pela Coordenação de Aperfeiçoamento de Pessoal de Nível Superior (CAPES) e pelo Conselho Nacional de Desenvolvimento Científico e Tecnológico (CNPq). por $\hat{\mathbf{s}}$, é disponível. Devido ao efeito do caminho secundário, o algoritmo FxLMS apresenta como desvantagem uma pobre característica de convergência. Para contornar tal inconveniente, uma modificação foi proposta em [3], resultando no algoritmo LMS filtrado modificado (MFxLMS). Esse algoritmo agora, através da remoção do efeito do caminho secundário, se comporta de forma similar ao LMS convencional [4], [5].

Para o projetista, é muito importante contar com um modelo estatístico para o algoritmo adaptativo estudado. Através desse modelo, é possível avaliar o comportamento do algoritmo, bem como identificar e ajustar os parâmetros críticos para se obter o melhor desempenho possível para uma dada aplicação. Muitos modelos estatísticos das diferentes versões do algoritmo LMS têm sido derivados sob a luz da teoria da independência (TI) [6], [7]. Entretanto, para se obter uma modelagem adequada dos algoritmos pertencentes à família de algoritmos de erro filtrado, a TI não pode mais ser invocada como mostrado em [8] e [9]. Assim, o objetivo deste trabalho é derivar um modelo analítico para o algoritmo MFxLMS, preenchendo assim uma lacuna nessa área de aplicação. Da mesma forma como em [8] e [9], o momento de primeira ordem do vetor de coeficientes do filtro adaptativo é obtido considerando sinais de entrada Gaussianos, sem invocar a TI. Por simplicidade matemática, o momento de segunda ordem é obtido levando em conta a condição de adaptação lenta. Resultados de simulação Monte Carlo (MC) ratificam a precisão do modelo proposto tanto para sinais de entrada brancos quanto coloridos.

Este trabalho é organizado como segue. A Seção II apresenta a derivação do modelo do algoritmo MFxLMS, iniciando com uma descrição do algoritmo FxLMS convencional, seguindo com sua versão modificada. Ainda nessa mesma seção, o modelo para o momento de primeira ordem do vetor de coeficientes e para a curva de aprendizagem são apresentados. Na Seção III, visando avaliar a precisão do modelo proposto, são mostrados resultados de simulações numéricas. Finalmente, a Seção IV apresenta alguns comentários e as conclusões finais deste trabalho.

\section{DerivaÇÃo do Modelo}

\section{A. Equação de Atualização dos Coeficientes para o Algoritmo FXLMS}

A Fig. 1 mostra o diagrama de blocos do algoritmo FxLMS convecional. A notação utilizada é a seguinte: $\mathbf{w}_{\mathrm{o}}=\left[\begin{array}{llll}w_{\mathrm{o}, 0} & w_{\mathrm{o}, 1} & \cdots & w_{\mathrm{o}, N-1}\end{array}\right]^{\mathrm{T}} \quad$ e $\quad \mathbf{w}(n)=\left[\begin{array}{lll}w_{0}(n) & w_{1}(n) & \cdots\end{array}\right.$ 
$\left.w_{N-1}(n)\right]^{\mathrm{T}}$ denotam os vetores de comprimento $N$ da planta e do filtro adaptativo, respectivamente. O caminho secundário é $\mathbf{s}=\left[\begin{array}{llll}s_{0} & s_{1} \cdots s_{M-1}\end{array}\right]^{\mathrm{T}}$ e sua estimativa, $\hat{\mathbf{s}}=\left[\begin{array}{llll}\hat{s}_{0} & \hat{s}_{1} \cdots & \hat{s}_{\hat{M}-1}\end{array}\right]^{\mathrm{T}}$. As variáveis $\mathbf{x}(n)=\left[\begin{array}{llll}x(n) & x(n-1) & \cdots & x(n-N+1)\end{array}\right]^{\mathrm{T}}$ e $d(n)$ são o vetor de entrada e o sinal desejado (ou primário), respectivamente. Neste trabalho, considera-se que o processo $\{x(n)\}$ é Gaussiano com variância $\sigma_{x}^{2}$. O sinal $z(n)$ é um ruído de medição de média zero com variância $\sigma_{z}^{2}$ e descorrelacionado de qualquer outro sinal no sistema. $\mathrm{O}$ vetor contendo o sinal de referência filtrado é $\mathbf{x}_{\mathrm{f}}(n)=\left[x_{\mathrm{f}}(n)\right.$ $\left.x_{\mathrm{f}}(n-1) \cdots x_{\mathrm{f}}(n-N-1)\right]^{\mathrm{T}}$, obtido a partir de

$$
\mathbf{x}_{\mathrm{f}}(n)=\sum_{i=0}^{\hat{M}-1} \hat{s}_{i} \mathbf{x}(n-i)
$$

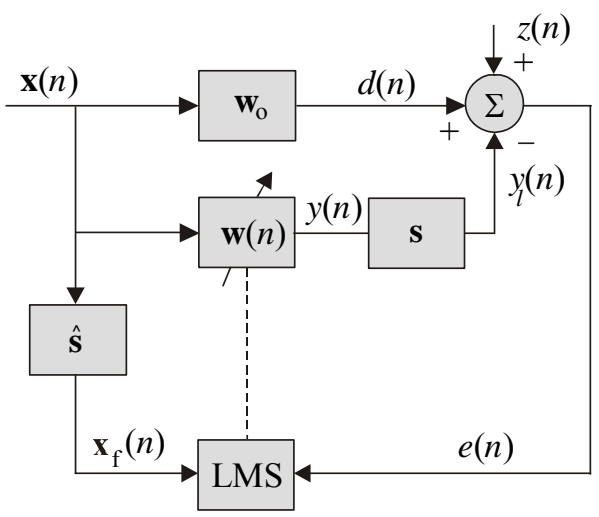

Fig. 1. Diagrama de bloco do algoritmo FxLMS.

Neste trabalho, considera-se que $\mathbf{w}_{\mathrm{o}}$ e $\mathbf{w}(n)$ têm a mesma dimensão; entretanto, pode não ser o caso para os vetores $\mathbf{s}$ e $\hat{\mathbf{s}}$. Assim, a modelagem proposta contempla dimensões diferentes para esses últimos vetores, como acontece em casos práticos.

Da Fig. 1 o sinal de erro é

$$
e(n)=d(n)-y_{l}(n)+z(n)
$$

com $d(n)$ e $y_{l}(n)$ dados, respectivamente, por

$$
d(n)=\mathbf{w}_{\mathrm{o}}^{\mathrm{T}} \mathbf{x}(n)=\mathbf{x}^{\mathrm{T}}(n) \mathbf{w}_{\mathrm{o}}
$$

e

$$
y_{l}(n)=\sum_{i=0}^{M-1} s_{i} \mathbf{w}^{\mathrm{T}}(n-i) \mathbf{x}(n-i) .
$$

Substituindo (4) em (2), obtém-se

$$
e(n)=d(n)-\sum_{i=0}^{M-1} s_{i} \mathbf{w}^{\mathrm{T}}(n-i) \mathbf{x}(n-i)+z(n) .
$$

Finalmente, a partir de (1) até (5), a expressão para a atualização do vetor de coeficientes do algoritmo FxLMS convencional é dada por [10]

$$
\mathbf{w}(n+1)=\mathbf{w}(n)+\mu e(n) \mathbf{x}_{\mathrm{f}}(n) .
$$

\section{B. Algoritmo MFxLMS}

O algoritmo MFxLMS é obtido a partir da introdução de um termo de compensação no sinal de erro instantâneo [equação (5)] [3]. Dessa forma, o erro compensado $\hat{e}(n)$ é

$$
\hat{e}(n)=d(n)-\sum_{i=0}^{M-1} s_{i} \mathbf{w}^{\mathrm{T}}(n-i) \mathbf{x}(n-i)+z(n)-\Lambda_{\mathrm{f}}(n)
$$

e a expressão para atualização dos coeficientes é então dada por

$$
\mathbf{w}(n+1)=\mathbf{w}(n)+\mu \hat{e}(n) \mathbf{x}_{\mathrm{f}}(n) .
$$

Em (7), a variável $-\Lambda_{\mathrm{f}}(n)$ denota o termo de compensação adicionado. Tal termo é determinado forçando que (7) tenha as mesmas características que o sinal de erro do algoritmo LMS convencional [3] [aqui denotado por $\left.\left.e(n)\right|_{\text {LMS }}\right]$, o qual depende dos valores atuais do vetor de coeficientes. Assim, o sinal de erro com características similares ao do algoritmo LMS convencional é dado por

$$
\left.e(n)\right|_{\mathrm{LMS}}=d(n)-\mathbf{w}^{\mathrm{T}}(n) \mathbf{x}_{\mathrm{f}}(n)+z(n) .
$$

Substituindo (1) em (9), tem-se

$$
\left.e(n)\right|_{\mathrm{LMS}}=d(n)-\sum_{i=0}^{M-1} s_{i} \mathbf{w}^{\mathrm{T}}(n) \mathbf{x}(n-i)+z(n) .
$$

Fazendo $\hat{e}(n)=\left.e(n)\right|_{\mathrm{LMS}}$, de (7) e (10), obtém-se $\Lambda_{\mathrm{f}}(n)$. Assim,

$$
\Lambda_{\mathrm{f}}(n)=\sum_{i=0}^{M-1} s_{i}\left[\mathbf{w}^{\mathrm{T}}(n)-\mathbf{w}^{\mathrm{T}}(n-i)\right] \mathbf{x}(n-i) .
$$

O termo $\left[\mathbf{w}^{\mathrm{T}}(n)-\mathbf{w}^{\mathrm{T}}(n-i)\right]$ é facilmente determinado de (8); assim, (11) pode ser reescrita como

$$
\Lambda_{\mathrm{f}}(n)=\mu \sum_{i=0}^{M-1} \sum_{j=1}^{i} s_{i} \hat{e}(n-j) \mathbf{x}_{\mathrm{f}}^{\mathrm{T}}(n-j) \mathbf{x}(n-i) .
$$

Finalmente, substituindo (12) em (7), obtém-se a expressão do erro compensado

$$
\begin{aligned}
\hat{e}(n)= & d(n)-\sum_{i=0}^{M-1} s_{i} \mathbf{w}^{\mathrm{T}}(n-i) \mathbf{x}(n-i) \\
& -\mu \sum_{i=0}^{M-1} \sum_{j=1}^{i} s_{i} \hat{e}(n-j) \mathbf{x}_{\mathrm{f}}^{\mathrm{T}}(n-j) \mathbf{x}(n-i)+z(n) .
\end{aligned}
$$


Note que (13) é uma expressão recursiva em $\hat{e}(n)$. É essa característica que faz com que o algoritmo modificado tenha um comportamento similar ao de um LMS convencional.

\section{Comportamento Médio do Vetor de Coeficientes}

Nesta seção, é obtido o momento de primeira ordem do vetor de coeficientes do algoritmo MFxLMS. Então, substituindo (1) e (13) em (8) e tomando o valor esperado de ambos os lados da expressão resultante, tem-se

$$
\begin{aligned}
& E[\mathbf{w}(n+1)]=E[\mathbf{w}(n)]+\mu \sum_{i=0}^{\hat{M}-1} \hat{s}_{i} E[\mathbf{x}(n-i) d(n)] \\
& -\mu \sum_{i=0}^{M-1} \sum_{k=0}^{\hat{M}-1} s_{i} \hat{s}_{k} E\left[\mathbf{x}(n-k) \mathbf{w}^{\mathrm{T}}(n-i) \mathbf{x}(n-i)\right] \\
& -\mu^{2} \sum_{i=0}^{M-1} \sum_{j=1}^{i} \sum_{k=0}^{\hat{M}^{-1}} s_{i} \hat{s}_{k} E\left[\mathbf{x}(n-k) \hat{e}(n-j) \mathbf{x}_{\mathrm{f}}^{\mathrm{T}}(n-j) \mathbf{x}(n-i)\right] \\
& +\mu \sum_{i=0}^{\hat{M}-1} \hat{s}_{i} E[\mathbf{x}(n-i) z(n)] .
\end{aligned}
$$

Agora, usando (1), (3) e (5) para determinar $\mathbf{x}_{\mathrm{f}}^{\mathrm{T}}(n-j), d(n)$ e $\hat{e}(n-j)$, respectivamente, e substituindo em (14), após simples manipulações matemáticas, obtém-se

$$
\begin{aligned}
& E[\mathbf{w}(n+1)]=E[\mathbf{w}(n)]+\mu \sum_{i=0}^{\hat{M}-1} \hat{s}_{i} \mathbf{R}_{-i} \mathbf{w}_{\mathrm{o}} \\
& -\mu \sum_{i=0}^{M-1} \sum_{k=0}^{\hat{M}-1} s_{i} \hat{s}_{k} \mathbf{R}_{i-k} E[\mathbf{w}(n-i)] \\
& -\mu^{2} \sum_{i=0}^{M-1} \sum_{j=1}^{i} \sum_{k=0}^{\hat{M}-1} \sum_{p=0}^{\hat{M}-1} s_{i} \hat{s}_{k} \hat{s}_{p}\left\{\mathbf{R}_{j+p-k} \mathbf{R}_{j-i}+\mathbf{R}_{i-k} \mathbf{R}_{-p}\right. \\
& \left.+\mathbf{R}_{j-k} \operatorname{tr}\left[\mathbf{R}_{j+p-i}^{\mathrm{T}}\right]\right\} \mathbf{w}_{\mathrm{o}} \\
& +\mu^{2} \sum_{i=0}^{M-1} \sum_{j=1}^{i} \sum_{k=0}^{\hat{M}-1} \sum_{l=0}^{M-1} \sum_{p=0}^{\hat{M}-1} s_{i} \hat{s}_{k} s_{l} \hat{s}_{p}\left\{\mathbf{R}_{j+p-k} \mathbf{R}_{j+l-i}\right. \\
& +\mathbf{R}_{i-k} \mathbf{R}_{l-p}+\mathbf{R}_{j+l-k} \operatorname{tr}\left[\mathbf{R}_{j+p-i}^{\mathrm{T}}\right] E[\mathbf{w}(n-j-l)] \\
& +\mu^{3} \sum_{i=0}^{M-1} \sum_{j=1}^{i} \sum_{k=0}^{\hat{M}-1} \sum_{l=0}^{M-1} \sum_{m=1}^{l} \sum_{p=0}^{\hat{M}-1} s_{i} \hat{s}_{k} s_{l} \hat{s}_{p} E[\mathbf{x}(n-k) \\
& \times \hat{e}(n-j-m) \mathbf{x}_{\mathrm{f}}^{\mathrm{T}}(n-j-m) \mathbf{x}(n-j-l) \\
& \left.\times \mathbf{x}^{\mathrm{T}}(n-j-p) \mathbf{x}(n-i)\right] \\
& -\mu^{2} \sum_{i=0}^{M-1} \sum_{j=1}^{i} \sum_{k=0}^{\hat{M}-1} \sum_{p=0}^{\hat{M}-1} s_{i} \hat{s}_{k} \hat{s}_{p} E[\mathbf{x}(n-k) z(n-j) \\
& \left.\times \mathbf{x}^{\mathrm{T}}(n-j-p) \mathbf{x}(n-i)\right]+\mu \sum_{i=0}^{\hat{M}-1} \hat{s}_{i} E[\mathbf{x}(n-i) z(n)] .
\end{aligned}
$$

Os valores esperados em (15) são determinados levando em conta as seguintes considerações de análise:

i) As correlações entre os diferentes vetores de entrada são muito mais importantes do que as correlações entre o vetor de entrada e o vetor de coeficientes [8].

ii) $\mathrm{O}$ modelo é derivado considerando adaptação lenta. Assim, em (15), os termos afetados por $\mu^{\beta}$ com $\beta \geq 3$ são desconsiderados.

iii) Dado que o sinal de entrada é Gaussiano, o momento de quarta ordem é determinado através do Teorema de Fatoração de Momentos para sinais Gaussianos [11].

iv) Todas as matrizes de autocorrelação em (15) são obtidas a partir da forma geral $\mathbf{R}_{\beta-\alpha}=E\left[\mathbf{x}(n-\alpha) \mathbf{x}^{\mathrm{T}}(n-\beta)\right]$.

Considerando (i) até (iv) e as características do ruído $z(n)$, pode-se reescrever (15) como segue:

$$
\begin{aligned}
& E[\mathbf{w}(n+1)]=E[\mathbf{w}(n)]+\mu \sum_{i=0}^{\hat{M}-1} \hat{s}_{i} \mathbf{R}_{-i} \mathbf{w}_{\mathrm{o}} \\
& -\mu \sum_{i=0}^{M-1} \sum_{k=0}^{\hat{M}-1} s_{i} \hat{s}_{k} \mathbf{R}_{i-k} E[\mathbf{w}(n-i)] \\
& -\mu^{2} \sum_{i=0}^{M-1} \sum_{j=1}^{i} \sum_{k=0}^{\hat{M}-1} \sum_{p=0}^{\hat{M}-1} s_{i} \hat{s}_{k} \hat{s}_{p}\left\{\mathbf{R}_{j+p-k} \mathbf{R}_{j-i}+\mathbf{R}_{i-k} \mathbf{R}_{-p}\right. \\
& \left.+\mathbf{R}_{j-k} \operatorname{tr}\left[\mathbf{R}_{j+p-i}^{\mathrm{T}}\right]\right\} \mathbf{w}_{\mathrm{o}}+\mu^{2} \sum_{i=0}^{M-1} \sum_{j=1}^{i} \sum_{k=0}^{M} \sum_{l=0}^{M-1} \sum_{p=0}^{M} s_{i} \hat{s}_{k} s_{l} \hat{s}_{p} \\
& \times\left\{\mathbf{R}_{j+p-k} \mathbf{R}_{j+l-i}+\mathbf{R}_{i-k} \mathbf{R}_{l-p}+\mathbf{R}_{j+l-k} \operatorname{tr}\left[\mathbf{R}_{j+p-i}^{\mathrm{T}}\right]\right. \\
& \times E[\mathbf{w}(n-j-l)]\} .
\end{aligned}
$$

\section{Vetor de Coeficientes em Regime Permanente}

Assumindo que o algoritmo converge, o valor de $\mathbf{w}(n) \mathrm{em}$ regime permanente, denotado por $\mathbf{w}_{\infty}$, é obtido a partir da seguinte condição:

$$
\begin{aligned}
\lim _{n \rightarrow \infty} E[\mathbf{w}(n+1)] & =\lim _{n \rightarrow \infty} E[\mathbf{w}(n-i)]=\lim _{n \rightarrow \infty} E[\mathbf{w}(n-j-l)] \\
& =\lim _{n \rightarrow \infty} E[\mathbf{w}(n)]=\mathbf{w}_{\infty} .
\end{aligned}
$$

Substituindo (17) em (16) e considerando um passo de adaptação pequeno, obtém-se

$$
\mathbf{w}_{\infty}=\sum_{i=0}^{M-1} \sum_{k=0}^{\hat{M}-1} \sum_{l=0}^{\hat{M}-1} s_{i} \hat{s}_{k} \hat{s}_{l} \mathbf{R}_{i-k}^{-1} \mathbf{R}_{-l} \mathbf{w}_{\mathrm{o}} .
$$

Então, de (18), verifica-se que na condição de adaptação lenta o algoritmo MFxLMS tem o mesmo comportamento de regime permanente que o algoritmo FxLMS dado em [8]. Em outras palavras, a modificação introduzida não afeta as características de regime permanente, modificando apenas o regime transiente do algoritmo. 


\section{E. Curva de Aprendizagem}

Para determinar a expressão da curva de aprendizagem, é utilizada a condição $\hat{e}(n)=\left.e(n)\right|_{\text {LMS }}$; assim, elevando-se ao quadrado ambos os lados de (10) e tomando o valor esperado de ambos os lados da expressão resultante, obtém-se

$$
\begin{aligned}
& E\left[\hat{e}^{2}(n)\right]=E\left[d^{2}(n)\right]-2 \sum_{i=0}^{M-1} s_{i} E\left[d(n) \mathbf{x}^{\mathrm{T}}(n-i) \mathbf{w}(n)\right] \\
& +\sum_{i=0}^{M-1} \sum_{j=0}^{M-1} s_{i} s_{j} E\left[\mathbf{w}^{\mathrm{T}}(n) \mathbf{x}(n-i) \mathbf{x}^{\mathrm{T}}(n-j) \mathbf{w}(n)\right]+E\left[z^{2}(n)\right] .
\end{aligned}
$$

Logo, considerando as características do ruído $z(n)$, usando (i)-(iv) e (3), tem-se

$$
\begin{aligned}
& \xi(n)=\mathbf{w}_{\mathrm{o}}^{\mathrm{T}} \mathbf{R}_{\mathrm{o}}-2 \mathbf{w}_{\mathrm{o}}^{\mathrm{T}} \sum_{i=0}^{M-1} s_{i} \mathbf{R}_{i} E[\mathbf{w}(n)] \\
& +\sum_{i=0}^{M-1} \sum_{j=0}^{M-1} s_{i} s_{j} \operatorname{tr}\left\{E\left[\mathbf{w}(n) \mathbf{w}^{\mathrm{T}}(n)\right] \mathbf{R}_{j-i}\right\}+\sigma_{z}^{2}
\end{aligned}
$$

onde $\xi(n)=E\left[\hat{e}^{2}(n)\right]$. Para finalizar a derivação de (20), necessita-se determinar o momento de segunda ordem do vetor de coeficientes. Assim, considerando a condição de adaptação lenta, o momento de segunda ordem requerido pode ser aproximado por $E\left[\mathbf{w}(n) \mathbf{w}^{\mathrm{T}}(n)\right] \cong$ $E[\mathbf{w}(n)] E\left[\mathbf{w}^{\mathrm{T}}(n)\right][10]$. Dessa forma, já temos disponível uma expressão para o valor esperado de $\mathbf{w}(n)$, tendo sido determinada em (16).

\section{F. Modelo Invocando a TI}

Para fins de comparação, também são determinadas as expressões do momento de primeira ordem e da curva de aprendizagem sob a luz da TI. Tal teoria estabelece que $\mathbf{R}_{\beta-\alpha}=E\left[\mathbf{x}(n-\alpha) \mathbf{x}^{\mathrm{T}}(n-\beta)\right]=0$ para $\alpha \neq \beta$. Portanto, a expressão do momento de primeira ordem considerando então a TI é dada por

$$
\begin{aligned}
& E[\mathbf{w}(n+1)]= \\
& E[\mathbf{w}(n)]+\mu \hat{s}_{0} \mathbf{R} \mathbf{w}_{\mathrm{o}}-\mu \sum_{i=0}^{\min (M, \hat{M})-1} s_{i} \hat{s}_{i} \mathbf{R} E[\mathbf{w}(n-i)] \\
& -\mu^{2} \sum_{i=0}^{M-1} \sum_{j=1}^{i} \sum_{k=0}^{\hat{M}-1} \sum_{p=0}^{\hat{M}-1} s_{i} \hat{s}_{k} \hat{s}_{p}\left\{\mathbf{R}_{1}+\mathbf{R}_{2}+\mathbf{R}_{3}\right\} \mathbf{w}_{\mathrm{o}} \\
& +\mu^{2} \sum_{i=0}^{M-1} \sum_{j=1}^{i} \sum_{k=0}^{\hat{M}-1} \sum_{l=0}^{M-1} \sum_{p=0}^{M-1} s_{i} \hat{s}_{k} s_{l} \hat{s}_{p}\left\{\mathbf{R}_{4}+\mathbf{R}_{5}+\mathbf{R}_{6}\right\} E[\mathbf{w}(n-j-l)]
\end{aligned}
$$

onde

$$
\begin{aligned}
& \mathbf{R}_{1}= \begin{cases}\mathbf{R}^{2}, & j+p=k \text { e } j=i \\
0, & \text { caso contrário }\end{cases} \\
& \mathbf{R}_{2}= \begin{cases}\mathbf{R}^{2}, & i=k \text { e } p=0 \\
0, & \text { caso contrário }\end{cases} \\
& \mathbf{R}_{3}= \begin{cases}\mathbf{R} \operatorname{tr}[\mathbf{R}], & j=k \text { e } j+p=i \\
0, & \text { caso contrário }\end{cases} \\
& \mathbf{R}_{4}= \begin{cases}\mathbf{R}^{2}, & j+p=k \text { e } j+l=i \\
0, & \text { caso contrário }\end{cases} \\
& \mathbf{R}_{5}= \begin{cases}\mathbf{R}^{2}, & i=k \text { e } l=q \\
0, & \text { caso contrário }\end{cases} \\
& \mathbf{R}_{6}= \begin{cases}\mathbf{R} \operatorname{tr}[\mathbf{R}], & j+l=k \text { e } j+p=i \\
0, & \text { caso contrário }\end{cases}
\end{aligned}
$$

Logo, a curva de aprendizagem obtida usando a TI é

$$
\begin{aligned}
\xi(n)= & \mathbf{w}_{\mathrm{o}}^{\mathrm{T}} \mathbf{R} \mathbf{w}_{\mathrm{o}}-2 \mathbf{w}_{\mathrm{o}}^{\mathrm{T}} s_{0} \mathbf{R} E[\mathbf{w}(n)] \\
& +\sum_{i=0}^{M-1} s_{i}^{2} \operatorname{tr}\left\{E\left[\mathbf{w}(n) \mathbf{w}^{\mathrm{T}}(n)\right] \mathbf{R}\right\}+\sigma_{z}^{2} .
\end{aligned}
$$

A partir de (21) e (22), observa-se que invocando a TI muitos dos termos são desconsiderados, levando o modelo a fornecer resultados inadequados. Na próxima seção, esses resultados serão melhor evidenciados.

\section{Resultados de SimulaÇÃo}

Nesta seção, dois exemplos são apresentados a fím de verificar a precisão do modelo proposto. O primeiro exemplo considera um sinal de entrada branco e o segundo, um sinal colorido. Sem perda de generalidade, para todos os exemplos é utilizada a condição $\mathbf{s}=\hat{\mathbf{s}}$.

\section{A. Exemplo 1}

Para este exemplo, a planta é dada por

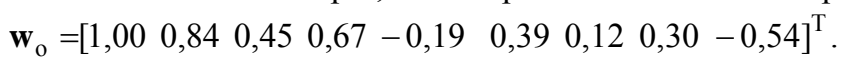
O caminho secundário é dado por $\mathbf{s}=\left[\begin{array}{llll}0,502 & 0,335 & 0,167\end{array}\right]^{\mathrm{T}}$. O sinal de entrada $x(n)$ é Gaussiano com $\sigma_{x}^{2}=1$. O máximo valor do passo de adaptação $\mu_{\max }$ (determinado experimentalmente) para o algoritmo é igual a 0,15 . Nesse exemplo, é utilizado um passo de adaptação $\mu=0,6 \mu_{\max }$. Os resultados de simulação Monte Carlo (MC) (média de 500 realizações independentes) e as predições obtidas a partir do modelo proposto [(16) e (20)] são mostrados na Fig. 2. Dessa figura, observa-se que o modelo proposto prediz satisfatoriamente o comportamento médio do vetor de coeficientes do filtro adaptativo como também a curva de aprendizagem (erro quadrático médio - EQM). Os resultados obtidos a partir do modelo considerando a TI [(21) e (22)] são também apresentados na Fig. 2, evidenciando o descasamento decorrente de tal modelo. 


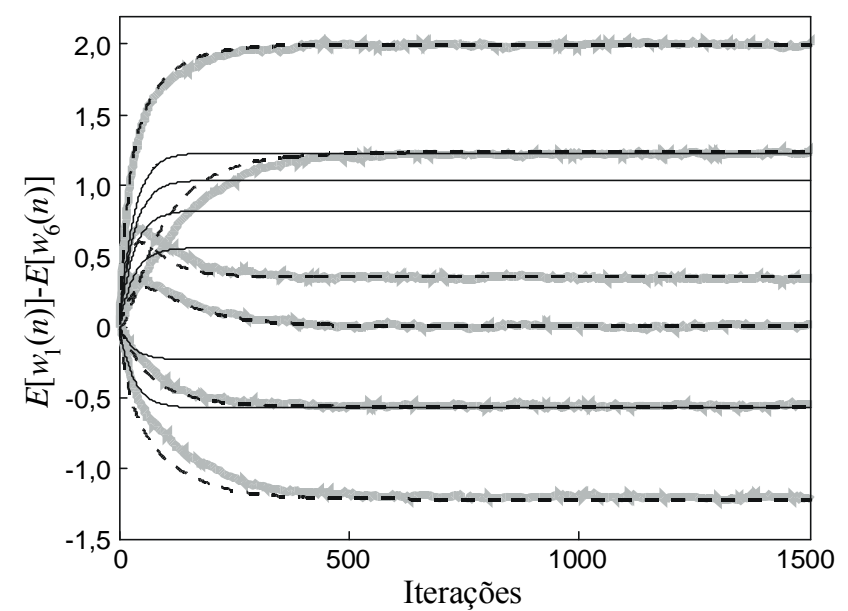

(a)

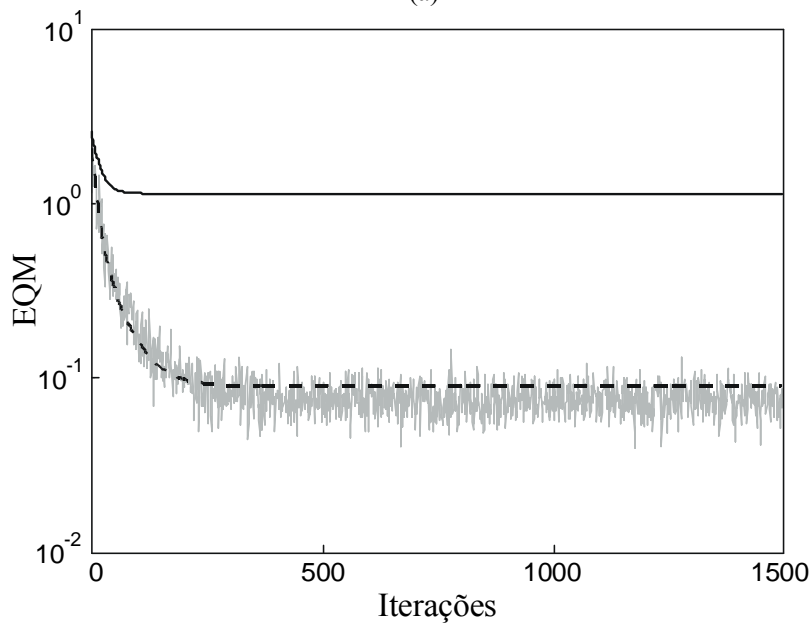

(b)

Fig. 2. Exemplo 1. Sinal de entrada branco e $\mu=0,6 \mu_{\max }$. Simulação $\mathrm{MC}$ (linha irregular cinza); modelo considerando a TI (linha contínua preta); modelo proposto (linha tracejada preta). (a) Comportamento médio do vetor de coeficientes. (b) Curva de aprendizagem.

\section{B. Exemplo 2}

Neste caso, é utilizado um sinal de entrada colorido, o qual é obtido através de um processo $\mathrm{AR}(2)$ dado por

$$
x(n)=a_{1} x(n-1)+a_{2} x(n-2)+u(n)
$$

onde $u(n)$ é um ruído branco com variância unitária. Os coeficientes do processo $\operatorname{AR}(2)$ são $a_{1}=0,1833$ e $a_{2}=-0,75$, resultando em uma dispersão dos autovalores da matriz de autocorrelação de entrada igual a 26,87. A planta e o caminho secundário são os mesmos utilizados no exemplo anterior. O máximo passo de adaptação para esse caso é $\mu_{\max }=0,2$ (determinado experimentalmente). Para este exemplo, é usado $\mu=0,6 \mu_{\max }$. As Figs. 3(a) e (b) mostram o momento de primeira ordem e as curvas de EQM dos modelos teóricos (usando a TI e o modelo proposto), respectivamente. Através dessas figuras, pode-se verificar que o modelo proposto [expressões (16) e (20)] apresenta um bom casamento com as simulações MC. Em contraste, o modelo derivado considerando a TI [(21) e (22)] não prediz adequadamente os resultados de simulação.

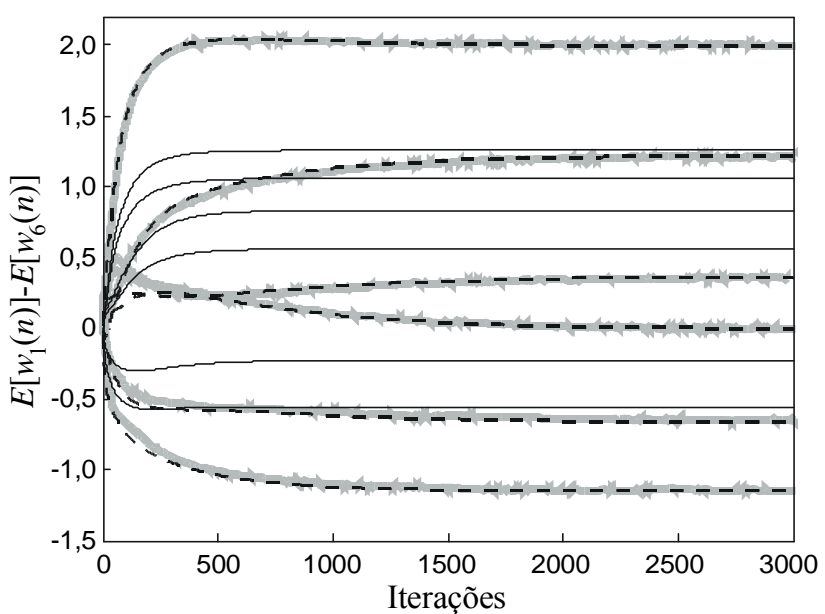

(a)

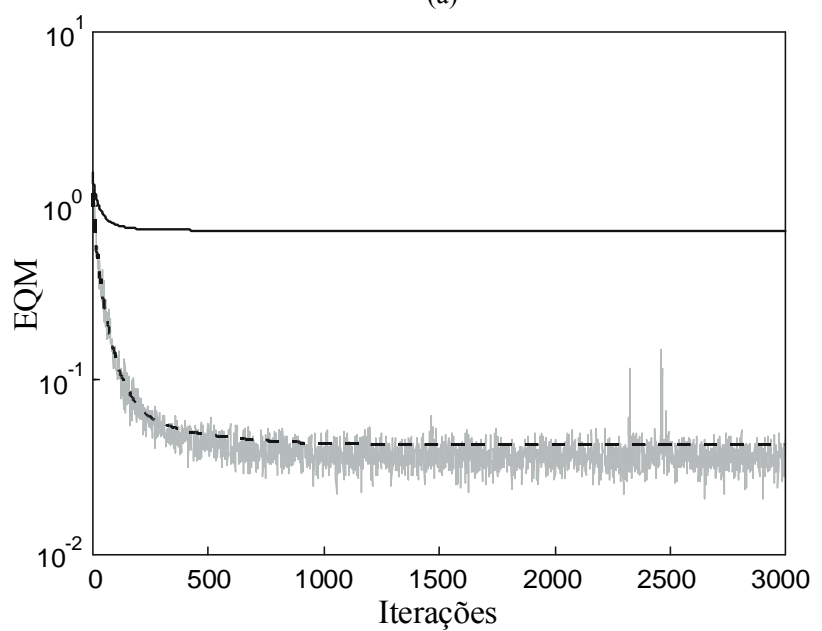

(b)

Fig. 3. Exemplo 2. Sinal de entrada colorido e $\mu=0,6 \mu_{\max }$. Simulação $\mathrm{MC}$ (linha irregular cinza); modelo considerando a TI (linha contínua preta); modelo proposto (linha tracejada preta). (a) Comportamento médio do vetor de coeficientes. (b) Curva de aprendizagem.

\section{CONCLusões}

Modelos analíticos para o momento de primeira ordem e da curva de aprendizagem do algoritmo MFxLMS foram determinados. Esses modelos são obtidos sem invocar a clássica teoria da independência e considerando uma condição de adaptação lenta. Simulações numéricas verificaram o bom desempenho do modelo proposto tanto para sinais de entrada Gaussianos brancos quanto coloridos.

\section{REFERÊNCIAS}

[1] S. J. Elliott and P. A. Nelson, "Active noise control," IEEE Signal Processing Mag., vol. 10, no. 4, pp. 12-35, Oct. 1993.

[2] J. R. Glover Jr., "Adaptive noise canceling applied to sinusoidal interferences," IEEE Trans. Acoust., Speech, Signal Process., vol. 25, no. 6, pp. 484-491, Dec. 1977.

[3] R. D. Poltman, "Conversion of the delayed LMS algorithm into the LMS algorithm," IEEE Signal Process. Lett., vol. 2, no. 12, pp. 223, Dec. 1995.

[4] S. C. Douglas, "An efficient implementation of the modified filtered-X LMS algorithm," IEEE Signal Process. Lett., vol. 4, no. 10, pp. 286-288, Oct. 1997.

[5] M. Rupp, "Saving complexity of modified filtered-X LMS and delayed update LMS algorithms," IEEE Trans. Circuits Syst. II: Anal. Dig. Signal Process., vol. 44, no. 1, pp. 45-48, Jan. 1997. 
[6] B. Widrow and S. D. Stearns, Adaptive Signal Processing, Prentice Hall, 1998.

[7] E. Bjarnason, "Analysis of the filtered-X LMS algorithm," IEEE Trans. Speech and Audio Process., vol. 3, no. 6, pp. 504-514, Nov. 1995.

[8] O. J. Tobias, J. C. M. Bermudez, N. J. Bershad, and R. Seara, "Mean weight behavior of the filtered-X LMS algorithm," in Proc. IEEE Int. Conf. Acoust., Speech, Signal Process., Seattle, USA, May 1998, pp. 3545-3548.

[9] - "Second moment analysis of the filtered-X LMS algorithm," in Proc. IEEE Int. Conf. Acoust., Speech, Signal Process., Phoenix, USA, Mar. 1999, pp. 1873-1876, .

[10] N. J. Bershad, P. Celka, and J. M. Vesin, "Stochastic analysis of gradient adaptive identification of nonlinear systems with memory for Gaussian data and noisy input and output measurements," IEEE Trans. Signal Processing, vol. 47, no. 3, pp. 675-689, Mar. 1999.

[11] B. Farhang-Boroujeny, Adaptive Filters: Theory and Applications, John Wiley \& Sons, 1998. 